PDFlib PLOP: PDF Linearization, Optimization, Protection

Page inserted by evaluation version www.pdflib.com - sales@pdflib.com 


\title{
Small Heat-Shock Protein Family: Function in Health and Disease
}

\author{
MICHAEL J. WELSH ${ }^{a, b}$ AND MATTHIAS GAESTEL ${ }^{a, c}$ \\ ${ }^{b}$ Department of Anatomy and Cell Biology, University of Michigan \\ Medical School, Ann Arbor, Michigan 48109 USA \\ 'Max-Delbrück Centre for Molecular Medicine, Robert-Rössle-Str. 10, \\ 13122 Berlin and Martin-Luther-University Halle-Wittenberg, \\ Innovationskolleg Zellspezialisierung, Hoher Weg 8, 06120 Halle, \\ Germany
}

\section{INTRODUCTION}

The following is a review of the small heat shock protein (sHsp) workshop (B1) held in Budapest on July 2, 1997 at the "Stress of Life" congress. Several excellent reports concerning sHsps were also presented at other workshops during the congress and will be included in this summary to the extent possible. New information about the structure and function of sHsps was given on the following topics.

\section{LARGE OLIGOMERS OF sHsPS}

One of the most characteristic features of all small heat-shock proteins described so far is their oligomerization to large intracellular aggregates (400-800 $\mathrm{kDa}$ or sometimes even larger). There are several data that indicate that phosphorylation of mammalian sHsps could influence its oligomeric size. sHsp phosphorylation was further analyzed, and for mouse Hsp25 and human Hsp27 it is clearly the result of an activated p38 MAP kinase cascade, and MAPKAP kinases 2 or 3 and can be inhibited by the compound SB203580 (J. Landry, Canada; M. Gaestel, Germany; Workshop A2). Interestingly, it was shown using a green fluorescent protein-MAPKAP kinase 2 fusion protein that MAPKAP kinase 2 is stored in its inactive form in the nucleus of the cell. During its activation by phosphorylation, however, it is rapidly exported to the cytoplasm where the sHsps are localized. This could probably represent a new mechanism of stress-dependent signal transduction increasing the specificity of the response (M. Gaestel). Phosphorylation of the sHsp $\alpha$ B-crystallin in U373 MG glioma cells was reported to be stimulated by several agents, such as, PMA, sodium arsenite, or ocadaic acid (K. Kato, Japan). The phosphorylation sites in $\alpha \mathrm{B}$-crystallin were determined to be S19, S45, and S59. Using phosphorylation site specific antibodies, it was shown that stress-induced phosphorylation of S59 but not PMA-induced phosphorylation of S45 could be inhibited by the p38 protein kinase inhibitor SB202190. This indicates that various protein kinases could phosphorylate this sHsp at different sites in response to certain extracellular stimuli (K. Kato).

${ }^{a}$ Additional correspondence information for Michael J. Welsh: Fax: 313-763-1166; e-mail: welsh@umich.edu

For Matthias Gaestel: Fax: 4930 94063798; e-mail: gaestel@mdc-berlin.de 
At the workshop, the oligomerization of phosphorylation mutants of sHsps was described. Molecular mimicry of serine phosphorylation by aspartate in human Hsp27 was reported to result in a decrease in the oligomer size of purified recombinant protein from nearly $800 \mathrm{kDa}$ to below $100 \mathrm{kDa}$ (M. Gaestel). Similarily, it was shown in workshop A2 that replacement of the phosphorylatable serines in hamster Hsp27 by glutamic acid decreases the molecular weight of the mutant oligomers when expressed in transfected cells (J. Landry). In both cases the phosphorylation of $\mathrm{S} 15$ in the $\mathrm{NH}_{2}$-terminal proline-phenylalanine-rich region of the sHsps ${ }^{1}$ seemed of special importance for disintegration of the large sHsp oligomers (J. Landry, M. Gaestel). In contrast, phosphorylation of the sHsp aB-crystallin did not change its degree of oligomerization (K. Kato). The workshop also provided several ideas concerning what a change of oligomerization of sHsps could mean at the functional level (see below).

\section{IN VITRO-CHAPERONE FUNCTION OF SHSPS}

As an attempt to better understand the in vitro-chaperone function of sHsps at the molecular level, NMR studies of $\alpha$-crystallin and its binding to unfolding proteins were presented (J. Carver and R. Lindner, Australia). From NMR spectra it could be concluded that sHsps are relatively flexible in the $\mathrm{COOH}$-terminal part of the proteins, including the last 8 to 10 amino acids of the crystallins and the last 18 amino acids of Hsp25. This flexible tail appears to be important for maintaining solubility of the protein oligomer. In studies of how large oligomers of $\alpha \mathrm{A}$ and $\alpha \mathrm{B}$-crystallin interact with unfolding proteins, J. Carver analyzed NMR spectra depicting changes in protein structure as heat or urea-denatured $\gamma$-crystallin (a protein that exists as a monomer) was diluted into a solution of large $\alpha \mathrm{A}$ - and $\alpha \mathrm{B}-$ crystallin oligomers. In this process, the NMR signal of the flexible, carboxyl tail of $\alpha \mathrm{B}$-crystallin was not seen, but the signal of the $\alpha \mathrm{A}$-crystallin tail remained. This result indicated that the tail of $\alpha B$-crystallin probably interacts with the $\gamma$ crystallin and that the $\alpha$ A tail remained free, probably to maintain solubility of the large complex.

In addition, the interaction of $\alpha$-lactalbumin with the large $\alpha$-crystallin oligomer was analyzed by NMR, and it was found that $\alpha$-crystallin interacts with unfolding proteins that are in the disordered molten globule state rather than proteins in an ordered molten globular state (R. Lindner). Thus, $\alpha$-crystallin seems to be involved with proteins after the proteins have left the normal folding and unfolding pathway, when they are in the process of irreversibly denaturing and aggregating. R. Lindner received the Hans-Selye Award for Young Researchers for the above presentation.

Further data that contribute to the understanding of the in vitro-chaperone properties of sHsp and that were obtained in collaboration with J. Buchner's group were described by M. Gaestel. The ability of Hsp25 to facilitate the reactivation of citrate synthase in vitro was analyzed. Citrate synthase was first heat inactivated in the presence of Hsp25, then after the temperature was lowered, the presence of Hsp25 in the mixture prevented aggregation and precipitation of the citrate synthase. Significant reactivation of the enzymic activity of the citrate synthase could subsequently be realized by the further addition to the mixture of ATP and Hsp70. Addition of ATP alone did not result in any reactivation of enzyme activity. These in vitro results were interpreted as supporting the concept that 
Hsp25 may function in vivo as a "buffer" for unfolding protein intermediates in cells after heat shock until Hsp70 can refold the proteins. ${ }^{2}$ It was also mentioned that analysis of the in vitro-chaperone properties of the desaggregated phosphorylation site mutants of Hsp27 was carried out and that the small oligomers (these were suggested to be tetramers or possibly even trimers) could not function to suppress thermal aggregation of citrate synthase (M. Gaestel and J. Buchner). It was suggested that the phosphorylation-dependent dissociation of large Hsp25 oligomers into small oligomers can be a mechanism used by cells to clear protein folding intermediates from Hsp25 oligomers (M. Gaestel).

\section{PROTECTION AGAINST OXIDATIVE STRESS}

Cells that normally express only low levels of sHsp were transfected to express either higher levels of wild type or phosphorylation site mutants of sHsps (P. Arrigo, France). It was shown that cells can be protected from oxidative stress resulting from treatment with TNF- $\alpha$ or hydrogen peroxide by expression of wild type sHsps or by the mutant in which alanines have replaced serines. The alanine mutant mimics the nonphosphorylated sHsp and remains in the large oligomeric state. In addition to protecting cells against oxidative stress, expression of $\mathrm{sHsp}$ blocked activation of the transcription factor NF- $\mathrm{BB}$ and reduced the cellular levels of free radicals, oxidized lipids, and oxidized proteins. Expression of sHsp also blocked apoptosis. ${ }^{3}$ The mechanism by which sHsp may cause all of the abovelisted effects is not yet known. However, results were presented showing that the protective effect of sHsps involves glutathione because depletion of glutathione by buthionine sulfoximine (BSO) resulted in loss of the protective effect and overexpression of sHsp increases intracellular gluthathione levels. ${ }^{4}$ Thus, Hsp25 appears to be an important regulator of apoptosis, with this activity depending on the oligomeric state of Hsp25, with glutathione-dependent protection requiring large Hsp25 oligomers. It is not known whether Hsp27 protection against all apoptosis pathways involves the glutathione-protecting effects of Hsp27. Glutathione appears to be essential for protection from TNF $\alpha$ /oxidative stress-triggered apoptosis, but it is not yet known if glutathione is required for Hsp27 protection against Fas- or staurosporine-induced apoptosis (Arrigo). These observations are of potentially great importance, and it seems certain that researchers from many disciplines will have increased interest in the small stress proteins as a consequence. It is important to emphasize, however, that although the phenomenology has been clearly demonstrated, as yet the precise mechanisms by which Hsp25 relates to glutathione and to apoptosis remain unknown. Consequently, it is easy to predict that considerable effort will be directed in the immediate future toward defining the mechanisms involved in these events.

\section{SMALL OLIGOMERS OF SHSPS}

Just as large oligomers of sHsps now appear to be the specific form of sHsps involved with chaperone activities, different events appear to specifically require small oligomers, or possibly even monomers of Hsp27 (although no evidence yet indicates that monomers exist in vivo). It has been known for some time that phos- 
phorylation of Hsp27 decreases its oligomer size (discussed above). Phosphorylation of Hsp27, by activation of p38 kinase and MAPKAP kinase 2/3, is also coincident with stabilization of microfilaments in cells (Landry, workshops A2 and E5). Increased stabilization of microfilaments occurred in cells expressing increased wild type Hsp27 (which can be phosphorylated); but in cells in which the serine sites of phosphorylation of Hsp27 had been mutated to glycines (which cannot be phosphorylated); or in cells treated with SB203580, little affect on microfilaments was observed. It was also reported (Landry, in workshop A2) that vascular endothelial growth factor (VEGF), an important angiogenic factor, or $\mathrm{H}_{2} \mathrm{O}_{2}$, a byproduct of inflammation, induce reorganization of $\mathrm{f}$-actin that was dependent on activation of p38 kinase and Hsp27 phosphorylation. As well, results were presented indicating that VEGF activation of $\mathrm{p} 38 / \mathrm{Hsp} 27$ pathways is required for cell migration, suggesting an important role for Hsp27- and actin-dependent events in angiogenic processes. Because the serines to glycines mutation, as well as SB203580 treatment, resulted in Hsp27 remaining in the large oligomer form, while phosphorylation of wild-type Hsp27 resulted in small oligomers, it was concluded that the active form of Hsp27 for the effect on microfilaments is small oligomers. Because the phosphorylation-dependent effects on microfilaments are also coincident with protection of cells against subsequent stress, during discussion a potential discrepancy became apparent between evidence for glutathionedependent protection of cells by large oligomers (Arrigo) and protection by small oligomers that is possibly mediated by microfilaments (Landry, in workshop E5). This raises the question: what are the similarities and/or differences in events involving glutathione-dependent protection of cells from apoptosis by large oligomers and protection of cells by small oligomers that is coincident with stabilization of microfilaments? Although no clear explanation yet exists, based on differences in experimental model systems employed by Arrigo and Landry, it was suggested that events related to glutathione-dependent protection and apoptosis may occur at low Hsp27 expression levels and that a different series of events involving stabilization of microfilaments and coincident cell protection occurs when Hsp27 is expressed at higher levels.

\section{BINDING REGIONS OF SHSPS}

Because oligomerisation of both Hsp27 and $\alpha$-crystallins occurs, there has been interest in defining the regions responsible for homomeric and heteromeric binding of the sHsps. It has been speculated for some time that a highly conserved region of the sHsps (amino acids 125-140 of Hsp27 and an identical sequence in crystallin) is responsible for binding of sHsps to each other. It was shown, using the yeast two-hybrid system, that the carboxyl half of $\alpha \mathrm{B}$-crystallin contains the domain responsible for binding of $\alpha \mathrm{B}$-crystallin to itself as well as to Hsp27 (Boelens, the Netherlands). Because it had been suggested previously that the sHsps might be involved in intermediate filament assembly, whether any of the mammalian sHsps could bind to the intermediate filament proteins vimentin or desmin was examined (Boelens). The two-hybrid system assay was negative for sHsps binding to either vimentin or desmin, although the assay was positive for vimentin to vimentin binding as well as desmin to desmin binding. It was noted, however, that if interaction between sHsps and intermediate filaments requires formation of oligomers of either protein, then the two-hybrid assay would probably not be capable of detecting such an interaction. The two-hybrid system was 
also used to examine Hsp27 and $\alpha \mathrm{B}$-crystallin binding to various portions of Hsp27 (M. Welsh, USA) and results compatible with Boelens' were obtained. It was found that the highly conserved sequence of the sHsps (amino acids 125-140 of Hsp27) did by itself have binding activity and that binding activity toward both $\alpha \mathrm{B}$-crystallin and Hsp27 was also present in the remaining carboxyl-terminal portion of Hsp27 (amino acids 140-206). Thus, an additional carboxyl-terminal sequence as well as the highly conserved region of amino acids 125-140 appears to be involved in sHsps interactions. No binding of the amino terminal region of Hsp27 (amino acids 1-124) to either Hsp27 or $\alpha \mathrm{B}$-crystallin was detected (Welsh). Whereas the two-hybrid system results may define dimer binding as occurring in the carboxyl-terminal region of sHsps, large oligomer formation may involve this site or other as-yet undefined regions of interaction.

\section{EXPRESSION AND LOCALIZATION OF SHSPS IN NORMAL AND DISEASED STATES}

Changes in expression of the sHsps can also play an important role in cell regulation. Patterns of Hsp27 expression in normal and ultraviolet (UV) lightinduced dermatitis (sunburn), as well as other skin pathologies were discussed (C. Bayerl, Germany). In normal skin, Hsp27 becomes significantly expressed in keratinocytes as they undergo late stages of differentiation during their transition from stratum spinosum to stratum granulosum. Thus, as in many other cell types, increased expression of Hsp27 is associated with cell differentiation. Why increased expression of Hsp27 is so widely correlated with cell differentiation remains to be determined. In UV dermatitis Hsp27 expression increased in less differentiated keratinocytes throughout the stratum spinosum; however, apoptotic "sunburn" cells were immunohistochemically negative for Hsp27. Merkel cells (neuroendocrine cells of the epidermis) and dermal nerve fibers also expressed more Hsp27 after UV exposure. Increases in Hsp27 seen after UV exposure may indicate induction of a stress response by cells as they recover from UV damage.

Information about changes in expression and localization of sHsps in cardiac and skeletal muscle was also presented (R. Benndorf, USA). In embryonic and neonatal rat heart both Hsp27 and $\alpha \mathrm{B}$-crystallin are highly expressed, but after birth levels of $\alpha \mathrm{B}$-crystallin remain constant while expression of Hsp27 decreases by $90 \%$. In diseased adult human heart, $\alpha \mathrm{B}$-crystallin levels change little, but Hsp27 expression is greatly increased, compared to normal adult human heart. ${ }^{5}$ This may be the result of the heart mounting a stress response to disease, or it may reflect a return to the fetal pattern of gene expression. Measurement of expression of other genes may decide which is the case.

Localization of sHsps in cardiac and skeletal muscle was particularly interesting (FIG. 1A). Both Hsp27 and $\alpha$ B-crystallin colocalize with actin filaments in Ibands and also in the M-lines of the sarcomeres. In diseased adult human heart, there appeared to be a reduction of sHsps in the I-bands and an increase at the edge of cells, suggesting a redistribution of protein (Benndorf). ${ }^{5}$ The functional significance of the colocalization of actin filaments and sHsps in muscle remains to be determined. Possibly, the stress proteins function to stabilize the actin filaments in sarcomeres; they may be involved in muscle cell development by assist- 


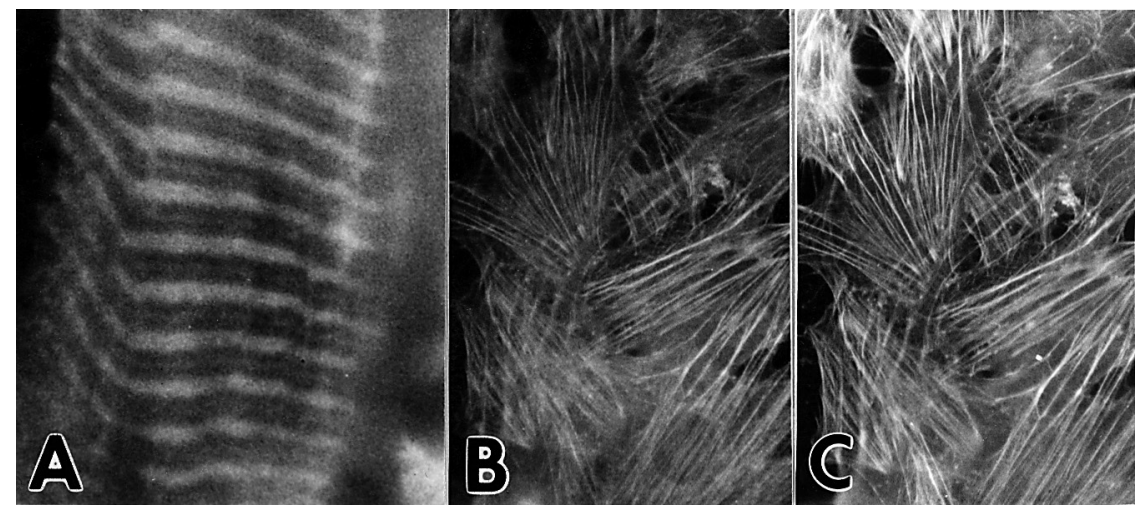

FIGURE 1. Subcellular localization of Hsp27 in a section of skeletal muscle and in cultured Sertoli cells. (A) Rat skeletal muscle showing immunofluorescence labeling of Hsp27. Hsp27 localizes to actin-containing I-bands. Hsp27-specific antibody used was from Stressgen (photo by Jill Pritts). (B) Immunofluorescence localization of Hsp27 in cultured rat Sertoli cells isolated from 27-day-old rats. Antibody was monoclonal 8A7 antibody. ${ }^{6}$ (C) Rodamine-phalloidin labeling of f-actin in the same Sertoli cells as are shown in panel B. Hsp27 and actin filaments co-localize in Sertoli cells.

ing in assembly of the highly ordered thin-filament arrays of the sarcomere, or they may even be involved in the mechanism of contraction.

\section{NOVEL HsP27-BINDING PROTEINS}

Localization of Hsp27 in Sertoli cells of the rat testis was also described (M. Welsh). In differentiated but not undifferentiated Sertoli cells, Hsp27 was colocalized with microfilaments ${ }^{6}$ (FIGs. 1B and 1C). It was proposed that this might be the result of expression only in differentiated Sertoli cells of a protein that mediates binding of Hsp27 to microfilaments. To investigate the hypothesis, a cDNA library was made from differentiated Sertoli cells and was used in the yeast two-hybrid system to identify potential Hsp27-binding proteins. In addition to identifying Hsp27, $\alpha \mathrm{B}$-crystallin and ubiquitin as Hsp27-binding proteins, two completely novel proteins were identified. The two proteins were expressed in E. coli as glutathione S-transferase (GST) fusion proteins and were used in an affinity chromatography procedure to determine whether Hsp27 and actin in cell homogenates could bind to the fusion proteins. Western blot analysis indicated that each of the binding proteins, but not GST alone, could bind Hsp27 as well as actin from cell homogenates (Welsh). These results support the hypothesis that either of these proteins might mediate Hsp27 association with microfilaments in vivo.

Some preliminary data was presented concerning the tissues that express the novel Hsp27-binding proteins. The mRNA for one of the novel Hsp27-binding proteins is expressed in many tissues, but the expression of the other Hsp27-binding protein may be restricted to fewer cell types. Northern blot analysis indicated that the second Hsp25-binding protein is highly expressed in purified Sertoli cells 
but much less so in whole testis (Welsh). That specific Hsp27-binding proteins exist and that some may exhibit cell-specific expression are exciting and novel observations that should contribute in a fundamental way to our concepts of Hsp27 function and potential mechanisms of action.

\section{SUMMARY}

In summary, several important issues were discussed at the workshop. These included the importance of oligomer size for different functions of the sHsps, the highly significant observation that the sHsps can block apoptosis; and that some mechanisms of sHsps protection involve glutathione while others may be related to microfilament stability. Further evidence was presented for the chaperone functions of sHsps, and structural studies provided additional information relating sHsps' large oligomeric structure to chaperone function. Regulation of oligomer size by phosphorylation was also a prominent topic of discussion, as was the importance of the relationship of oligomer size to differing functions of Hsp27. An interesting contrast was noted between Hsp27, whose oligomer size is regulated by phosphorylation, and $\alpha \mathrm{B}$-crystallin, whose large oligomer structure is unaffected by phosphorylation. This may prove to be of physiological significance, particularly in cell types that express both of these proteins. Observations of the translocation of MAPKAP kinase 2 from the nucleus to the cytoplasm in live cells suggest that the dynamics of phosphorylation of the sHsps may be more complex than previously thought. One topic that seems to have been settled is that the binding site of at least dimers of sHsps resides in the carboxyl-terminal region of the proteins. Ever-increasing numbers of studies are reporting interesting patterns of sHsp expression, phosphorylation, and subcellular localizations of the sHsps in various cells or tissues in normal and diseased states. Unfortunately, very little is understood about what these observations may mean. It can be expected, however, that as more is learned about the function and regulation of sHsps, the relationships between sHsps and cellular response to disease will become better understood. Lastly, the discovery that specific Hsp27-binding proteins may exist should open completely new avenues of investigation into the functions of sHsps. If the observations of unique subcellular localizations of sHsps in various cells types (e.g., striated muscle and Sertoli cells) can be related to expression of specific Hsp-binding proteins, considerable advances in our understanding of sHsps should result. It was abundantly clear from results presented and from ensuing discussions in the workshop that the study of sHsps is an exceedingly dynamic area of research (FIG. 2) with an ever-expanding significance for a number of other areas of current biological research. 


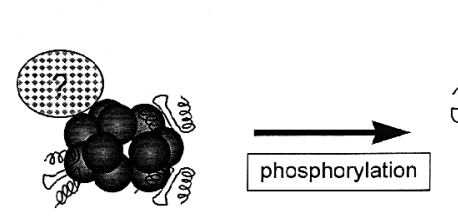

Chaperoning,

Protection against oxidative stress, Apoptosis

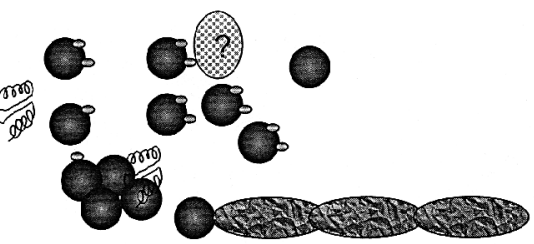

Organisation of the microfilaments

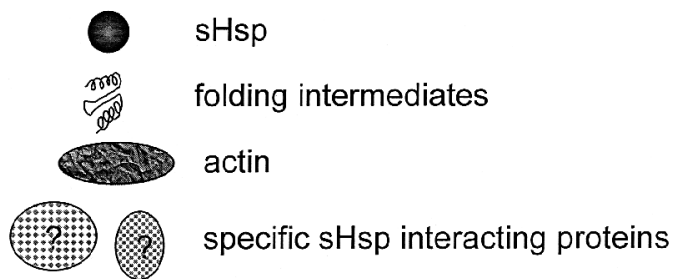

FIGURE 2. Hypothetical model for oligomerization-dependent functions of the sHsps. As large oligomers, sHsps chaperone intermediates of unfolding proteins and protect against oxidative stress and apoptosis. Small oligomers of sHsps influence organization of the microfilaments. The transition between the different oligomeric states is regulated by phosphorylation. Probably, other proteins (other chaperones or specific binding protein) mediate additional functions of sHsps.

\section{REFERENCES}

1. Ehrnsperger, M., M. GAESTEl \& J. BuCHNER. 1997. Structure and function of small heat shock proteins. In Molecular Chaperones in Proteins: Structure, Function and Mode of Action. A. Fink \& Y. Goto, Eds.: 533-575. Marcel Dekker, New York.

2. EHRNSPERGER, M., et al. 1997. Binding of non-native protein to Hsp25 during heat shock creates a reservoir of folding intermediates for reactivation. EMBO J. 16: 221-229.

3. MeHLEN-P., et al. 1996. Human hsp27, Drosophila hsp27 and human alphaB-crystallin expression-mediated increase in glutathione is essential for the protective activity of these proteins against TNFalpha-induced cell death. EMBO J. 15: 2695-2706.

4. Mehlen-P., K. SCHUlze-Osthoff \& A.-P. ARrigo. 1996. Small stress proteins as novel regulators of apoptosis. Heat shock protein 27 blocks Fas/APO-1- and staurosporineinduced cell death. J. Biol. Chem. 271: 16510-16514.

5. LuTSCH, G., et al. 1997. Abundance and location of the small heat shock proteins hsp25 and $\alpha B-c r y s t a l l i n$ in rat and human heart. Circulation 96: 3466-3476.

6. Welsh, M.J. et al. 1996. Variation in expression of hsp27 messenger ribonucleic acid during the cycle of the seminiferous epithelium and co-localization of hsp27 and microfilaments in Sertoli cells of the rat. Biol. Reprod. 55: 141-151. 